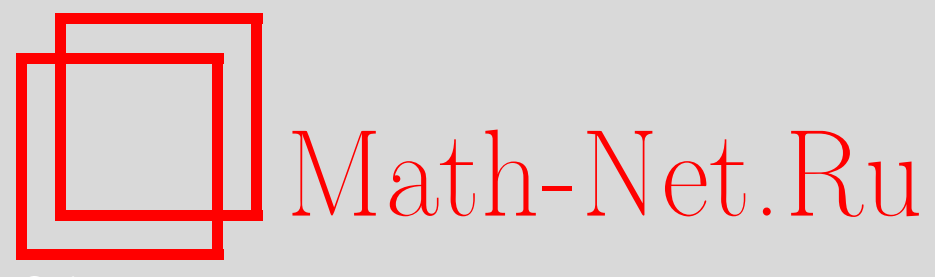

А. В. Чашкин, О сложности сужений булевых функций, Дискрет. матем., 1996, том 8, выпуск 2, 133-150

DOI: https://doi.org/10.4213/dm517

Использование Общероссийского математического портала Math-Net.Ru подразумевает, что вы прочитали и согласны с пользовательским соглашением http://www . mathnet.ru/rus/agreement

Параметры загрузки:

IP : 107.22 .136 .117

26 апреля 2023 г., 12:42:58

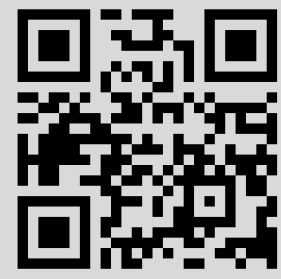




\title{
О сложности сужений булевых функций
}

\author{
(C) 1996 г. A. B. Чашкин
}

\begin{abstract}
Исследуется сложность сужений булевых функций при их реализации схемами из функциональных әлементов, контактными схемами, формулами и $\pi$ схемами. Пусть $D(d)=\left\{D \subseteq\{0,1\}^{n}|| D \mid=d\right\}$. Для достаточно широкого множества значений параметра $d$ для произвольной булевой функции $f$ устанавливаются нижние оценки сложности самого сложного ее сужения на области из $D(d)$. Обсуждается связь между сложностью частичных и полностью определенных булевых функций.

Работа выполнена при поддержке Российского фонда фундаментальных исследований, проект 93-011-1527.
\end{abstract}

\section{Введение}

Пусть $f\left(x_{1}, \ldots, x_{n}\right)$ - некоторая булева функция. Сложностью $L(f)$ булевой функции $f$ назовем сложность минимальной схемы, реализующей эту функцию в базисе $\{\&, \vee,-\}$. Сложностью $L_{k c}(f)$ булевой функции $f$ назовем сложность минимальной контактной схемы, реализующей $f$. Сложностью $L_{\phi}(f)$ булевой функции $f$ назовем сложность минимальной формулы, реализующей $f$ в базисе из всех не более чем двухместных булевых функций. Сложностью $L_{\pi}(f)$ булевой функции $f$ назовем сложность минимальной $\pi$-схемы, реализующей $f$. Пусть $D_{1} \subset D_{2} \subseteq\{0,1\}^{n}$. Функцию $g: D_{1} \rightarrow\{0,1\}$ назовем сужением функции $h: D_{2} \rightarrow$ $\{0,1\}$ на область $D_{1}$, если $g(x)=h(x)$ при любом $x \in D_{1}$. Сужение функции $f$ на область $D$ будем обозначать через $f_{D}$, т.е. для функций $g$ и $h$ справедливо равенство $g=h_{D_{1}}$.

В работе для упомянутых выше мер сложности исследуется величина самого сложного сужения произвольной булевой функции среди всех возможных сужений этой функции на области фиксированной мощности. Основная цель работы заключается в выявлении общих свойств, характерных как для относительно несложных функций, так и для функций, имеющих экспоненциальную сложность. В работе показано, что одним из таких общих свойств является сложность сужений функций, т.е. у любой функции, чья сложность превосходит некоторое пороговое значение, обязательно найдется очень сложное сужение. В связи с этим можно говорить о локальной “одинаковости" почти всех булевых функций.

В первом пункте работы исследуется сложность сужений булевых функций при их реализации схемами в базисе $\{\&, \mathrm{~V},-\}$. Во втором пункте рассмотрен частный случай: найдены точные по порядку значения сложности самых сложных сужений функции с малым числом единиц на области различной мощности. Результаты этого пункта позволяют получить информацию о точности 
неравенств, доказанных в первом пункте. В третьем пункте работы исследуются сложности сужений булевых функций при реализации функций $\pi$-схемами. Результаты, полученные в этом пункте, без труда могут быть перенесены на контактные схемы и формулы. В четвертом пункте работы обсуждается связь между сложностью частичных булевых функций и сложностью полностью определенных булевых функций. В этом пункте показано, что доказательство невысоких нижних оценок сложности частичных функций, определенных на областях малой мощности, и доказательство экспоненциальных нижних оценок сложности полностью определенных булевых функций являются в некотором смысле эквивалентными по трудности решения задачами. Результаты этого пункта в сочетании с результатами пунктов 1, 3 позволяют высказать предположение о том, что причины, не позволяющие в настоящее время доказывать даже сравнительно невысокие нижние оценки сложности булевых функций, имеют много общего с причинами, не позволяющими доказывать экспоненциальные нижние оценки сложности. Завершает работу добавление, в котором доказывается ряд результатов, необходимых в предыдущих пунктах.

\section{1. Общий случай}

Будем полагать, что $n$, число переменных всех рассматриваемых ниже функций, больше некоторой положительной постоянной. Пусть $D \subseteq\{0,1\}^{n}$, $f: D \rightarrow\{0,1\}, N(L, n)$ - число неизоморфных схем в базисе $\{\&, \vee,-\}$, имеющих $n$ входов, один выход и сложность, не превосходящую $L$. Если рассматривать $f$ как полностью определенную функцию, то можно утверждать, что для любого $d$, большего $(1+o(1)) \log _{2} N(L(f), n)$, существует такое сужение функции $f$ на некоторую область $D^{\prime},\left|D^{\prime}\right|=d$, что его сложность равна сложности $f$. Поэтому представляется естественным ограничить снизу мощности рассматриваемых областей сужения произвольной булевой функции $f$ величиной $\log _{2} N(L(f), n)$. В теореме 1 устанавливается нижняя оценка сложности самого сложного сужения произвольной функции $f$ на область фиксированной мошности $d$.

Теорема 1. Пусть $f:\{0,1\}^{n} \rightarrow\{0,1\}$, и $f$ существенно зависит от всех своих переменных. Тогда для любого $d$, где $\log _{2} N(L(f), n) \leqslant d \leqslant 2^{n}$, существуют тахие постоянная $c_{1}$ и область $D \in\{0,1\}^{n}$, что

$$
\begin{aligned}
|D| & \leqslant d, \\
L\left(f_{D}\right) & \geqslant \max \left\{n-1, c_{1} L(f)\left(\frac{1}{n} \log _{2} \frac{d n}{\log _{2} N(L(f), n)}\right)^{\log _{2} 3}\right\} .
\end{aligned}
$$

Доказательство теоремы основано на следующей лемме.

Лемма 1. Пусть

$$
D \subseteq\{0,1\}^{n}, \quad f: D \rightarrow\{0,1\}, \quad L(f) \geqslant n, \quad|D| \geqslant 2 \log _{2} N((L(f)-4) / 3, n) .
$$

Тогда существует такая область $D^{\prime} \subseteq D$, что

$$
\begin{aligned}
\left|D^{\prime}\right| & \leqslant 2\left(2|D| \log _{2} N((L(f)-4) / 3, n)\right)^{1 / 2}, \\
L\left(f_{D^{\prime}}\right) & \geqslant(L(f)-4) / 3 .
\end{aligned}
$$


Доказательство. Положим

$$
d=\left[\left(2|D| \log _{2} N((L(f)-4) / 3, n)\right)^{1 / 2}\right] .
$$

Рассмотрим множество областей

$$
M_{d}=\left\{V_{i}\left|V_{i} \subseteq D,\right| V_{i} \mid=d\right\}
$$

а также сужения функции $f$ на области этого множества. Возможны два случая:

- среди областей $V_{i} \in M_{d}$ существует область $V_{j}$, сложность сужения функции $f$ на которую удовлетворяет неравенству

$$
L\left(f_{V_{j}}\right) \geqslant(L(f)-4) / 3
$$

- сложность сужения функции $f$ на любую область из множества $M_{d}$ меньше чем $(L(f)-4) / 3$.

В первом случае утверждение леммы очевидно. В качестве $D^{\prime}$ достаточно взять область $V_{j}$. Рассмотрим второй случай. Так как $\left|M_{d}\right|=\left(\begin{array}{c}|D| \\ d\end{array}\right)$, и для любого целого $i, 1 \leqslant i \leqslant\left(\begin{array}{c}|D| \\ d\end{array}\right)$, сложность $L\left(f_{V_{i}}\right)$ не превосходит величины $(L(f)-4) / 3$, то существует схема $S$, сложность которой не больше, чем

$$
(L(f)-4) / 3
$$

и которая реализует на области $D$ некоторую булеву функцию $h$, совпадающую с функцией $f$ не менее чем на $\left(\begin{array}{c}|D| \\ d\end{array}\right) / N((L(f)-4) / 3, n)$ областях множества $M_{d}$. Пусть $V=D \backslash\left(\bigcup V_{i}\right)$, где объединение берется по всем областям $V_{i}$, на которых $f=h$. Оценим сверху мощность $R$ области $V$. Очевидно, что $R$ должна удовлетворять неравенству

$$
\frac{1}{N}\left(\begin{array}{c}
|D| \\
d
\end{array}\right)((L(f)-4) / 3, n) \leqslant\left(\begin{array}{c}
|D|-R \\
d
\end{array}\right)
$$

или, что равносильно, неравенству

$$
\left(\begin{array}{c}
|D| \\
d
\end{array}\right) /\left(\begin{array}{c}
|D|-R \\
d
\end{array}\right) \leqslant 2^{\log _{2} N((L(f)-4) / 3, n)} .
$$

В силу выбора параметра $d$ и условия леммы $|D| \geqslant 2 \log _{2} N((L(f)-4) / 3, n)$, можно воспользоваться леммой 6 , доказанной в добавлении.

Так как $2<e$, из леммы 6 и неравенства (5) следует, что

$$
R \leqslant d
$$

Далее, пусть

$$
d^{\prime}=\left[\left(2(|D|-R) \log _{2} N((L(f)-4) / 3, n)\right)^{1 / 2}\right] .
$$

Рассмотрим новое множество областей

$$
M_{d^{\prime}}=\left\{V_{i}^{\prime}\left|V_{i}^{\prime} \subseteq D, V \subset V_{i}^{\prime},\right| V_{i} \mid=d^{\prime}+R\right\}
$$

Как и ранее, возможны два случая: 
- среди областей $V_{i}^{\prime} \in M_{d^{\prime}}$ существует область $V_{j}^{\prime}$, сложность сужения функции $f$ на которую удовлетворяет неравенству

$$
L\left(f_{V_{j}^{\prime}}\right) \geqslant(L(f)-4) / 3
$$

- сложность сужения функции $f$ на любую область из множества $M_{d^{\prime}}$ меньше чем $(L(f)-4) / 3$.

В первом случае в качестве $D^{\prime}$ можно взять область $V_{j}^{\prime}$, так как в силу (6) $\left|V_{j}^{\prime}\right| \leqslant 2 d$. Если имеет место второй случай, то вновь воспользуемся леммой 6 . Рассуждая аналогично, нетрудно показать, что в этом случае существует некоторая булева функция $g$, определенная на области $D$, имеющая сложность не более, чем

$$
(L(f)-4) / 3
$$

и совпадающая с функцией $f$ не менее чем на $|D|-R^{\prime}, R^{\prime} \leqslant d^{\prime}$, наборах области $D$. Пусть эти наборы составляют область $W$, и пусть

$$
V^{\prime}=D \backslash W, \quad V^{\prime} \cap V=\varnothing .
$$

Рассмотрим далее такую функцию $z: D \rightarrow\{0,1\}$, значения которой при любом $x \in V \cup V^{\prime}$ совпадают с соответствующими значениями функции $f$. Нетрудно убедиться в справедливости равенства

$$
f=h g \vee h z \vee g z=h(g \vee z) \vee g z .
$$

Поэтому

$$
L(f) \leqslant L(h)+L(g)+L(z)+4,
$$

или, учитывая неравенства (4) и (7),

$$
L(f) \leqslant 2 L(f) / 3+4 / 3+L(z) .
$$

Из последнего неравенства и определения функции $z$ следует, что

$$
L\left(f_{V \cup V^{\prime}}\right)=L(z) \geqslant(L(f)-4) / 3 .
$$

Так как $\left|V \cup V^{\prime}\right| \leqslant 2 d$, лемма 1 доказана.

Доказательство теоремы 1. Применим к функции $f$ лемму 1. В результате получим область $V_{1} \subseteq D$ и сужение $f_{V_{1}}$ функции $f$ на эту область. К полученному сужению вновь применим лемму 1 и т.д. Предположим, что возможно последовательное применение леммы $1 s$ раз к соответствующим сужениям. Тогда получим последовательность вложенных областей

$$
V_{s} \subseteq V_{s-1} \subseteq \ldots \subseteq V_{1} \subseteq V_{0}=\{0,1\}^{n}
$$

и последовательность сужений функции $f$ на эти области. Из леммы 1 следует, что

$$
\begin{aligned}
\left|V_{i}\right| & \leqslant 2\left(2\left|V_{i-1}\right| \log _{2}\left(N\left(\left(L\left(f_{V_{i-1}}\right)-4\right) / 3\right)\right)\right)^{1 / 2} \\
L\left(f_{V_{i}}\right) & \geqslant\left(L\left(f_{V_{i-1}}\right)-4\right) / 3 .
\end{aligned}
$$


Так как $L\left(f_{V_{i}}\right) \leqslant L(f)$, оценку (8) можно заменить более слабой оценкой

$$
\left|V_{i}\right| \leqslant 2\left(2\left|V_{i-1}\right| \log _{2} N(L(f), n)\right)^{1 / 2} .
$$

Далее рассмотрим два случая:

(I) $d \geqslant n \log _{2} N(L(f), n)$,

(II) $d<n \log _{2} N(L(f), n)$.

Пусть имеет место первый случай. Оценим значение величины $2^{t}$ для минимального $t$, при котором $\left|V_{t}\right| \leqslant d$. Используем неравенство (10). Так как $\left|V_{t-1}\right|>d$, то

$$
d<\left|V_{t-1}\right| \leqslant 4\left(2^{n}\right)^{1 / 2^{t-1}} \log _{2} N(L(f), n) .
$$

Из последнего неравенства следует, что

$$
2^{t}<\frac{2 n}{\log _{2} \frac{d}{4 \log _{2} N(L(f), n)}}
$$

или, учитывая условие (I),

$$
2^{t} \leqslant c_{2} \frac{n}{\log _{2} \frac{d n}{\log _{2} N(L(f), n)}}
$$

где $c_{2}$ - некоторая постоянная. Очевидно, что при условии (I) неравенство (11) доказывает теорему, так как

$$
\begin{aligned}
\left|V_{t}\right| & \leqslant d \\
L\left(f_{V_{t}}\right) & \geqslant L(f)\left(\frac{1}{c_{2} n} \log _{2} \frac{d n}{\log _{2} N(L(f), n)}\right)^{\log _{2} 3}-6 .
\end{aligned}
$$

Теперь рассмотрим второй случай. Пусть $d^{\prime}=n \log _{2} N(L(f), n)$. Величина $d^{\prime}$ удовлетворяет условию первого случая. Следовательно, существуют такие область $D^{\prime}$ и сужение функции $f$ на $D^{\prime}$, что

$$
\begin{aligned}
\left|D^{\prime}\right| & \leqslant d^{\prime}, \\
L\left(f_{D^{\prime}}\right) & \geqslant c_{3} L(f)\left(\frac{\log _{2} n}{n}\right)^{\log _{2} 3},
\end{aligned}
$$

где $c_{3}-$ постоянная. Будем далее полагать, что

$$
L\left(f_{D^{\prime}}\right) \leqslant \frac{1}{n} L(f)
$$

Если последнее неравенство неверно, то очевидно, что существует область $D^{\prime \prime} \subseteq$ $D^{\prime}$, сложность сужения функции $f_{D^{\prime}}$ на которую удовлетворяет неравенству

$$
L\left(f_{D^{\prime \prime}}\right) \leqslant \frac{1}{n} L(f)
$$


Пусть минимальное значение $t$, при котором $\left|V_{t}\right| \leqslant d^{\prime}$, равно $t_{0}$. Оценим значение величины $2^{t_{0}+t_{1}}$ при условии, что

$$
\left|V_{t_{0}+t_{1}}\right| \leqslant d, \quad\left|V_{t_{0}+t_{1}-1}\right|>d .
$$

Из (8) и (13) следует неравенство

$$
\left|V_{t_{0}+t_{1}-1}\right| \leqslant 2\left(n \log _{2} N(L(f), n)^{1 / 2^{t_{1}-1}} \log _{2}(N(L(f) / n, n))^{1-1 / 2^{t_{1}-1}} .\right.
$$

В силу леммы 7 , доказанной в добавлении,

$$
\log _{2} N(L(f) / n, n) \leqslant \frac{1}{n-2} \log _{2} N(L(f), n) .
$$

Следовательно,

$$
\left|V_{t_{0}+t_{1}-1}\right| \leqslant 5 n^{-1+1 / 2^{t_{1}-2}} \log _{2} N(L(f), n) .
$$

Так как $\left|V_{t_{0}+t_{1}-1}\right|>d$, то

$$
2^{t_{1}}<c_{4} \frac{\log _{2} n}{\log _{2} \frac{d n}{\log _{2} N(L(f), n)}} \leqslant c_{4},
$$

где $c_{4}$ - некоторая постоянная. Объединяя неравенства (12) и (14), видим, что при некоторой постоянной $c_{5}$

$$
L\left(f_{V_{t_{0}+t_{1}}}\right) \geqslant c_{5} L(f)\left(\frac{\log _{2} n}{c_{4} n}\right)^{\log _{2} 3} .
$$

Неравенство (15) доказано в предположении, что лемма 1 может быть использована не менее чем $t_{0}+t_{1}$ раз. Пусть это не так, т.е. для некоторого $t^{\prime}$, меньшего чем $t_{0}+t_{1}$, лемма 1 не применима к функции $f_{V_{t^{\prime}}}$. Тогда либо

$$
L\left(f_{V_{t^{\prime}}}\right)<n
$$

либо

$$
\left|V_{t^{\prime}}\right|<2 \log _{2} N\left(\left(L\left(f_{V_{t^{\prime}}}\right)-4\right) / 3, n\right) .
$$

Если $L\left(f_{V_{t^{\prime}}}\right)<n$, то область $D$, сужение функции $f$ на которую существенно зависит от всех $n$ переменных, удовлетворяет условиям теоремы, так как

$$
|D| \leqslant 2 n, \quad L\left(f_{D}\right) \geqslant(n-1) \geqslant L\left(f_{V_{t^{\prime}}}\right) .
$$

Если

$$
\left|V_{t^{\prime}}\right|<2 \log _{2} N\left(\left(L\left(f_{V_{t^{\prime}}}\right)-4\right) / 3, n\right)
$$

то в силу леммы 7

$$
\left|V_{t^{\prime}}\right|<2 \log _{2} N\left(\left(L\left(f_{V_{t^{\prime}}}\right)-4\right) / 3, n\right) \leqslant \frac{2}{3} \log _{2} N\left(L\left(f_{V_{t^{\prime}}}\right), n\right)+\frac{2}{3}
$$

Пришли к противоречию. Следовательно, неравенство (16) невозможно. Теорема доказана. 
Результат приводимой ниже теоремы 2 неформально может быть сформулирован в виде следующего предложения. Функция $f$, сложность которой удовлетворяет неравенству

$$
L(f) \geqslant c_{6} n^{1+\log _{2} 3},
$$

обладает таким нетривиальным сужением $f_{D}$ на некоторую область $D$, что сложность әтого сужения только в некоторое постоянное число раз меньше сложности самой сложной частичной функции, определенной на $D$. Из теоремы 2 следует, что все функции, сложность которых больше, чем $c_{6} n^{1+\log _{2} 3}$, локально устроены одинаково: все такие функции имеют очень сложные сужения.

Теорема 2. Пусть

$$
f:\{0,1\}^{n} \rightarrow\{0,1\}, \quad L(f) \geqslant c_{6} n^{\log _{2} 3+1},
$$

где $c_{6}$ - постоянная. Тогда существуют тахие область $D \subseteq\{0,1\}^{n}$ и постоянная $c_{7}$, чmo

$$
L\left(f_{D}\right) \geqslant n, \quad \frac{|D|}{\log _{2} N\left(L\left(f_{D}\right), n\right)} \leqslant c_{7} .
$$

Доказательство теоремы основано на следующей лемме.

Лемма 2. Пусть

$$
D \subseteq\{0,1\}^{n}, \quad f: D \rightarrow\{0,1\}, \quad L(f) \geqslant n, \quad|D| \geqslant 2 \log _{2} N((L(f)-4) / 3, n) .
$$

Tогда сущестөуют тахие область $D^{\prime} \subseteq D$ и постоянная $c_{8}$, ито

$$
\frac{\left|D^{\prime}\right|}{\log _{2} N\left(L\left(f_{D^{\prime}}\right), n\right)} \leqslant c_{8}\left(\frac{|D|}{\log _{2} N(L(f), n)}\right)^{1 / 2} .
$$

Дохазатељьстөо. Пусть $D^{\prime}$ - область, доставляемая леммой 1. Тогда

$$
\begin{aligned}
\frac{\left|D^{\prime}\right|}{\log _{2} N\left(L\left(f_{D^{\prime}}\right), n\right)} & \leqslant \frac{2\left(2|D| \log _{2} N((L(f)-4) / 3, n)\right)^{1 / 2}}{\log _{2} N((L(f)-4) / 3, n)} \\
& =2 \sqrt{2}\left(\frac{|D|}{\log _{2} N((L(f)-4) / 3, n)}\right)^{1 / 2} .
\end{aligned}
$$

Нетрудно показать, что

$$
\log _{2} N((L(f)-4) / 3, n) \geqslant \frac{1}{4} \log _{2} N(L(f), n) .
$$

Подставляя последнее неравенство в (18), видим, что

$$
\frac{\left|D^{\prime}\right|}{\log _{2} N\left(L\left(f_{D^{\prime}}\right), n\right)} \leqslant 4 \sqrt{2}\left(\frac{|D|}{\log _{2} N(L(f), n)}\right)^{1 / 2} .
$$

Лемма доказана. 
Доказательство теоремы 2. Будем применять к функции $f$ лемму 2, пока это возможно. В результате применения леммы 2 получим последовательность вложенных областей $V_{i}$. Легко видеть, что

$$
\frac{\left|V_{i}\right|}{\log _{2} N\left(L\left(f_{V_{i}}\right), n\right)} \leqslant c_{8}^{2}\left(\frac{2^{n}}{\log _{2} N(L(f), n)}\right)^{1 / 2^{i}} .
$$

Предположим, что лемму 2 удалось применить $k$ раз. Тогда лемма 2 не может быть применена следующий $(k+1)$-й раз, если нарушено одно из условий леммы:

(I) $L\left(f_{V_{k}}\right) \geqslant n$,

(II) $\left|V_{k}\right| \geqslant 2 \log _{2} N\left(\left(L\left(f_{V_{k}}\right)-4\right) / 3, n\right)$.

Если $(k+1)$-му применению леммы 2 препятствует второе условие, то очевидно, что теорема доказана, так как в этом случае

$$
\frac{\left|V_{k}\right|}{\log _{2} N\left(L\left(f_{V_{k}}\right), n\right)}<2 .
$$

Теперь покажем, что если для $k-1$ справедливо условие (II), то справедливо и неравенство

$$
L\left(f_{V_{k}}\right) \geqslant n,
$$

из которого легко следует справедливость условия (I) для $k-1$. Пусть

$$
\begin{gathered}
\left|V_{k-1}\right| \geqslant 2 \log _{2} N\left(\left(L\left(f_{V_{k-1}}\right)-4\right) / 3, n\right), \\
\frac{\left|V_{k-1}\right|}{\log _{2} N\left(L\left(f_{V_{k-1}}\right), n\right)}<c_{8}^{2}\left(\frac{2^{n}}{\log _{2} N(L(f), n)}\right)^{1 / 2^{k-1}} .
\end{gathered}
$$

Объединив два последние неравенства, видим, что

$$
\begin{aligned}
2 \log _{2} N\left(\left(L\left(f_{V_{k-1}}\right)-4\right) / 3, n\right) \leqslant & \left|V_{k-1}\right| \\
& \leqslant c_{8}^{2}\left(\frac{2^{n}}{\log _{2} N(L(f), n)}\right)^{1 / 2^{k-1}} \log _{2} N\left(L\left(f_{V_{k-1}}\right), n\right) .
\end{aligned}
$$

Следовательно, учитывая (19), находим, что существует такая постоянная $c_{9}$, что

и поэтому

$$
\left(\frac{2^{n}}{\log _{2} N(L(f), n)}\right)^{1 / 2^{k}} \geqslant c_{9}
$$

Так как

$$
\left(\frac{1}{2}\right)^{k} \geqslant \frac{\log _{2} c_{9}}{\log _{2} \frac{2^{n}}{\log _{2} N(L(f), n)} .}
$$

$$
L\left(f_{V_{i}}\right) \geqslant\left(L\left(f_{V_{i-1}}\right)-4\right) / 3
$$

справедливы оценки

$$
L\left(f_{V_{k}}\right) \geqslant\left(\frac{1}{2}\right)^{k \log _{2} 3} L(f)-6 \geqslant c_{10}\left(\frac{1}{n}\right)^{\log _{2} 3} L(f) \geqslant n,
$$

где $c_{10}$ - постоянная. Неравенство (20), а вместе с ним и теорема 2, доказаны. 


\section{2. Функция с малым числом единиц}

Пусть $D \subseteq\{0,1\}^{n}, f: D \rightarrow\{0,1\}$. Весом функции $f$ назовем мощность множества $\{x \in D \mid f(x)=1\}$. Вес функции $f$ обозначим через $\|f\|$. Пусть $\alpha$ - постоянная, и пусть $1<\alpha<2$. Рассмотрим функцию $f^{\alpha}:\{0,1\}^{n} \rightarrow\{0,1\}$ веса $n^{\alpha}$, для сложности которой справедливо равенство

$$
L\left(f^{\alpha}\right)=\max _{\|f\| \leqslant n^{\alpha}} L(f)
$$

Из теоремы 3.4 из [1] следует, что

$$
L\left(f^{\alpha}\right)=c_{11} \frac{n^{1+\alpha}}{\log _{2} n}(1+o(1)),
$$

где $c_{11}$ - некоторая постоянная. Там же (лемма Д.3) найдена оценка числа неизоморфных схем в произвольном конечном базисе, имеющих $n$ входов, $m$ выходов и сложность которых не превосходит $L$. Из этой оценки легко извлекается следующая лемма.

Лемма 3. Справедлизо неравенство

$$
N(L, n) \leqslant\left(c_{12}(L+n)\right)^{L}
$$

где $c_{12}$ - некоторая постоянная.

Из (21) и леммы 3 следует существование такой постоянной $c_{13}$, что

$$
\log _{2}\left(N\left(L\left(f^{\alpha}\right), n\right)\right) \leqslant c_{13} n^{1+\alpha} .
$$

Пусть

$$
D(d)=\left\{D \subseteq\{0,1\}^{n}|| D \mid=d\right\}
$$

Теорема 3. Существуют такие постоянные $c_{14} u c_{15}$, что для любого $d$, $n^{1+\alpha} \leqslant d \leqslant 2^{n}$,

(1) для любой области $D \in D(d)$ справедлива оченка

$$
L\left(f_{D}^{\alpha}\right) \leqslant c_{14} \frac{n^{\alpha} \ln d}{\ln n}
$$

(2) существует такая область $D^{\prime} \in D(d)$, что

$$
L\left(f_{D^{\prime}}^{\alpha}\right) \geqslant c_{15} \frac{n^{\alpha} \ln d}{\ln n} .
$$

Доказательство первого предложения теоремы следует непосредственно из леммы 8, доказанной в добавлении.

Доказательство второго предложения теоремы основано на следующей лемме. 
Лємма 4. Пусть $D \subseteq\{0,1\}^{n}, f: D \rightarrow\{0,1\},\|f\|=t, L(f) \geqslant n$, $|D| \geqslant t+2 \log _{2} N(L(f) / 2-1, n)$. Tогда существует maxas область $D^{\prime} \subseteq D$, что

$$
\begin{gathered}
\left|D^{\prime}\right|-t \leqslant\left(2(|D|-t) \log _{2} N(L(f) / 2-1, n)\right)^{1 / 2}, \\
L\left(f_{D^{\prime}}\right) \geqslant(L(f)-1) / 2, \\
\left\|f_{D^{\prime}}\right\|=t .
\end{gathered}
$$

Доказательстөо. Настоящая лемма доказывается так же, как и лемма 1. Положим

$$
d=\left[\left(2(|D|-t) \log _{2} N((L(f)-1) / 2, n)\right)^{1 / 2}\right] .
$$

Рассмотрим множество областей

$$
M_{d}=\left\{V_{i}\left|V_{i} \subseteq D,\right| V_{i} \mid=d+t,\left\|f_{V_{i}}^{\alpha}\right\|=t\right\},
$$

а также сужения функции $f^{\alpha}$ на области әтого множества. Возможны два случая:

(I) среди областей $V_{i} \in M_{d}$ существует область $V_{j}$, сложность сужения функции $f^{\alpha}$ на которую удовлетворяет неравенству

$$
L\left(f_{V_{j}}^{\alpha}\right) \geqslant\left(L\left(f^{\alpha}\right)-1\right) / 2,
$$

(II) сложность сужения функции $f^{\alpha}$ на любую область из множества $M_{d}$ меньше, чем $\left(L\left(f^{\alpha}\right)-1\right) / 2$.

В первом случае утверждение леммы очевидно. Второй случай аналогичен случаю (II), рассмотренному при доказательстве леммы 1 . Нетрудно показать существование такой функции $h: D \rightarrow\{0,1\}$, совпадающей с функцией $f^{\alpha}$ на некоторой области $V$, что

$$
|D \backslash V| \leqslant d, \quad L(h)<(L(f)-1) / 2 .
$$

Рассмотрим теперь функцию $g: D \rightarrow\{0,1\}$, совпадающую с функцией $f^{\alpha}$ на области

$$
V^{\prime}=(D \backslash V) \cup\left\{x \mid f^{\alpha}(x)=1\right\} .
$$

Легко видеть; что $f^{\alpha}=h g$. Следовательно,

$$
L\left(f_{V^{\prime}}^{\alpha}\right)=L(g) \geqslant L\left(f^{\alpha}\right)-L(h)-1 \geqslant\left(L\left(f^{\alpha}\right)-1\right) / 2 .
$$

Так как $\left|V^{\prime}\right| \leqslant d+t$, то лемма доказана.

Перейдем непосредственно к доказательству второго предложения теоремы. Предположим, что к функции $f^{\alpha}$ возможно $s$ раз применить лемму 4 . В результате получим такую последовательность областей

$$
V_{s} \subseteq V_{s-1} \subseteq \ldots \subseteq V_{1} \subseteq V_{0}=\{0,1\}^{n},
$$

что при $1 \leqslant i \leqslant s$ 
(I) $\left|V_{i}\right|-n^{\alpha} \leqslant\left(2\left(\left|V_{i-1}\right|-n^{\alpha}\right) \log _{2} N\left(\left(L\left(f_{V_{i-1}}^{\alpha}\right)-1\right) / 2, n\right)\right)^{1 / 2}$,

(II) $L\left(f_{V_{i}}^{\alpha}\right) \geqslant\left(L\left(f_{V_{i-1}}^{\alpha}\right)-1\right) / 2$,

(III) $\left\|f_{V_{i}}^{\alpha}\right\|=n^{\alpha}$.

Полагая $L\left(f_{V_{i-1}}^{\alpha}\right)=L\left(f^{\alpha}\right)$, оценим величину $\left|V_{i}\right|-n^{\alpha}$. Ясно, что

$$
\left|V_{i}\right|-n^{\alpha} \leqslant\left(2\left(\left|V_{i-1}\right|-n^{\alpha}\right) \log _{2} N\left(L\left(f^{\alpha}\right), n\right)\right)^{1 / 2} \leqslant c_{16}\left(2^{n}\right)^{1 / 2^{i}} n^{1+\alpha}
$$

где $c_{16}$ - некоторая постоянная. Из последнего неравенства найдем минимальное $m, 1 \leqslant m \leqslant s$, при котором

$$
\left|V_{m}\right|-n^{\alpha} \leqslant d n^{3}-n^{\alpha}
$$

Так как

$$
d n^{3}-n^{\alpha}<\left|V_{m-1}\right|-n^{\alpha} \leqslant c_{13}\left(2^{n}\right)^{1 / 2^{m-1}} n^{1+\alpha}
$$

справедлива оценка

$$
m \leqslant \log _{2} n-\log _{2} \log _{2}(d n)+1 .
$$

Из леммы 8 следует неравенство

$$
L\left(f_{V_{m}}^{\alpha}\right) \leqslant c_{11} \frac{n^{\alpha} \ln d}{\ln n} .
$$

Заметим, что с другой стороны,

$$
L\left(f_{V_{m}}^{\alpha}\right) \geqslant\left(\frac{1}{2}\right)^{m} L(f)-1 \geqslant \frac{\log _{2} d n}{n} L(f)-1 \geqslant c_{17} \frac{\log _{2} d n}{n} L(f),
$$

где $c_{17}$ - постоянная.

Используя (23), оценим значение величины $2^{k}$ для минимального $k$, при котором

$$
\left|V_{m+k}\right|-n^{\alpha} \leqslant d-n^{\alpha} .
$$

Так как из (22), (23) следует, что при некоторых постоянных $c_{18}, c_{19}$

$$
\begin{aligned}
d / 2< & d-n^{\alpha}<\left|V_{m+k-1}\right|-n^{\alpha} \\
& \leqslant\left(d n^{3}-n^{\alpha}\right)^{1 / 2^{k-1}} c_{18} \frac{n^{\alpha} \log _{2} d}{\log _{2} n}\left(\log _{2} n+\log _{2} \log _{2} d\right) \leqslant c_{19}\left(d n^{3}\right)^{1 / 2^{k-1}} n^{\alpha} \log _{2} d,
\end{aligned}
$$

To

$$
2^{k-1} \leqslant \frac{\log _{2} d n^{3}}{\log _{2} \frac{d}{2 c_{19} n^{\alpha} \log _{2} d}} \leqslant \frac{8 \log _{2} d}{\log _{2} d}=8
$$

и, следовательно, учитывая (21) и (24),

$$
L\left(f_{V_{m+k}}^{\alpha}\right) \geqslant \frac{1}{16} L\left(f_{V_{m}^{2}}^{\alpha}\right) \geqslant \frac{c_{17} \log _{2} d n}{16 n} L\left(f^{\alpha}\right) \geqslant c_{15} \frac{n^{\alpha} \ln d}{\ln n} .
$$

Так как $d>n^{1+\alpha}$, то, очевидно, что при всех $i \leqslant m+k$

$$
L\left(f_{V_{i}}^{\alpha}\right) \geqslant c_{20} n^{\alpha}>n .
$$


Теперь для доказательства теоремы достаточно показать, что при всех $i \leqslant m+k$

$$
\left|V_{i}\right| \geqslant n^{\alpha}+2 \log _{2} N\left(\left(L\left(f_{V_{i}}^{\alpha}\right)-1\right) / 2, n\right) \text {. }
$$

Предположим, что это не так. Тогда из леммы 3 и леммы 7 следует, что

$$
\begin{aligned}
\left|V_{i}\right| & <n^{\alpha}+2 \log _{2} N\left(\left(L\left(f_{V_{i}}^{\alpha}\right)-1\right) / 2, n\right) \leqslant n^{\alpha}+L\left(f_{V_{i}}^{\alpha}\right) \log _{2} c_{12} L\left(f_{V_{i}}^{\alpha}\right) \\
& \leqslant 3 L\left(f_{V_{i}}^{\alpha}\right) \log _{2} L\left(f_{V_{i}}^{\alpha}\right) \leqslant c_{21} n^{\alpha} \ln V_{i} .
\end{aligned}
$$

Поэтому

$$
\frac{\left|V_{i}\right|}{\ln \left|V_{i}\right|}<c_{21} n^{\alpha}
$$

Так как величина, стоящая в левой части последнего неравенства, монотонно возрастает по $\left|V_{i}\right|$, а $\left|V_{i}\right| \geqslant n^{1+\alpha}$, то неравенство

$$
\frac{n^{1+\alpha}}{(1+\alpha) \ln n}<c_{21} n^{\alpha}
$$

приводит к противоречию со сделанным выше предположением. Теорема доказана.

\section{3. Контактные схемы, $\pi$-схемы и формулы}

Пусть $N_{\text {кс }}(L, n)$ равно числу неизоморфных двухполюсных контактных схем, которые имеют не более $L$ ребер, не содержат изолированных вершин и содержат только контакты из множества $\left\{x_{1}, \ldots, x_{n}, \bar{x}_{1}, \ldots, \bar{x}_{n}\right\}$. Пусть, далее, $N_{\pi}(L, n)$ равно числу неизоморфных $\pi$-схем, которые имеют не более $L$ ребер и содержат контакты из множества $\left\{x_{1}, \ldots, x_{n}, \bar{x}_{1}, \ldots, \bar{x}_{n}\right\}$, а $N_{\phi}(L, n)$ равно числу неизоморфных формул в базисе из всех не более чем двуместных булевых функций, которые содержат не более $L$ символов переменных из множества $\left\{x_{1}, \ldots, x_{n}\right\}$.

Результаты, аналогичные доказанным в теореме 1 и теореме 2 для схем из функциональных әлементов, имеют место и при реализации булевых функций контактными схемами, $\pi$-схемами и формулами в базисе из всех не более чем двуместных булевых функций. Причем формулировки и доказательства этих результатов для всех трех управляющих систем почти дословно повторяют друг друга. Поэтому ограничимся тем, что явно сформулируем эти результаты только для $\pi$-схем. Формулировки для контактных схем и формул отличаются от приводимых ниже формулировок для $\pi$-схем только функциями $L_{\text {кс }}, L_{\phi}, N_{\text {кс }}, N_{\phi}$, используемыми вместо функций $L_{\pi}$ и $N_{\pi}$.

Так как $\pi$-схемы изоморфны формулам в базисе $\{\&, \vee,-\}$, то результаты теорем 4, 5 справедливы также и для әтих формул.

Теорема 4. Пусть $f:\{0,1\}^{n} \rightarrow\{0,1\}$, u $f$ существенно зависит от өсех сөоих переменяых. Тогда для либого $d$, где

$$
\log _{2} N_{\pi}\left(L_{\pi}(f), n\right) \leqslant d \leqslant 2^{n},
$$

существуют тахие постоянная $c_{21}$ и область $D \subseteq\{0,1\}^{n}$, что

$$
|D| \leqslant d, \quad L_{\pi}\left(f_{D}\right) \geqslant \max \left(n, c_{21} L_{\pi}(f)\left(\frac{1}{n} \log _{2} \frac{d n}{\log _{2} N_{\pi}\left(L_{\pi}(f), n\right)}\right)^{2}\right) .
$$


Доказательство теоремы 4 выводится из следующей леммы так же, как и доказательство теоремы 1 выводится из леммы 1 . Поэтому доказательство теоремы 4 опустим, ограничившись доказательством леммы 5.

Лемма 5. Пусть

$$
D \subseteq\{0,1\}^{n}, \quad f: D \rightarrow\{0,1\}, \quad L_{\pi}(f) \geqslant n, \quad|D| \geqslant 2 \log _{2} N_{\pi}\left(L_{\pi}(f) / 2, n\right) .
$$

Тогда существует такая область $D^{\prime} \subseteq D$, ито

$$
\left|D^{\prime}\right| \leqslant 2\left(2|D| \log _{2} N_{\pi}\left(L_{\pi}(f) / 2, n\right)\right), \quad L_{\pi}\left(f_{D^{\prime}}\right) \geqslant L(f) / 2 .
$$

Доказательство. Пусть

$$
d=\left[\left(2\|f\| \log _{2} N_{\pi}\left(L_{\pi}(f) / 2, n\right)\right)^{1 / 2}\right] .
$$

Рассмотрим множество областей

$$
M_{d}=\left\{V_{i}\left|V_{i} \subseteq D,\{x \mid x \in D, f(x)=0\} \subseteq V_{i},\right| V_{i} \mid=\|\bar{f}\|+d\right\},
$$

а также сужения функции $f$ на области этого множества. Как и при доказательстве леммы 4, можно показать, что существует такая область $V \subseteq D$, что

(I) $|V|-\|\bar{f}\| \leqslant\left(2(|D|-\|\bar{f}\|) \log _{2} N_{\pi}\left(L_{\pi}(f) / 2, n\right)\right)^{1 / 2}$;

(II) $L_{\pi}\left(f_{V}\right) \geqslant L_{\pi}(f) / 2$;

(III) $\left\|f_{V}\right\|=|V|-\|\bar{f}\|$.

Далее, положим

$$
d^{\prime}=\left[\left(2\left\|\bar{f}_{V}\right\| \log _{2} N_{\pi}\left(L_{\pi}\left(f_{V}\right) / 2, n\right)\right)^{1 / 2}\right]
$$

Рассмотрим множество областей

$$
M_{d^{\prime}}=\left\{V_{i}^{\prime}\left|V_{i}^{\prime} \subseteq V,\left\{x \mid x \in V, f_{V}(x)=1\right\} \subseteq V_{i}^{\prime},\right| V_{i}^{\prime} \mid=\left\|f_{V}\right\|+d^{\prime}\right\},
$$

а также сужения функции $f$ на области этого множества. Как и ранее нетрудно показать, что существует такая область $V^{\prime} \subseteq V$, что

$\left(I^{\prime}\right)\left|V^{\prime}\right|-\left\|f_{V}\right\| \leqslant\left(2\left(|V|-\left\|f_{V}\right\|\right) \log _{2}^{*} N_{\pi}\left(L_{\pi}\left(f_{V}\right) / 2, n\right)\right)^{1 / 2}$

(II') $L_{\pi}\left(f_{V^{\prime}}\right) \geqslant L_{\pi}(f) / 2$.

Из (II) и (II') следует неравенство

$$
L_{\pi}\left(f_{V^{\prime}}\right) \geqslant L_{\pi}(f) / 4
$$

Из (III), (I') и леммы 5 следует, что

$$
\begin{aligned}
\left|V^{\prime}\right| & \leqslant\left\|f_{V}\right\|+\left(2\left(|V|-\| f_{V}\right) \| \log _{2} N_{\pi}\left(L_{\pi}\left(f_{V}\right) / 2, n\right)\right)^{1 / 2} \\
& \leqslant|V|-\|\bar{f}\|+\left(2\left(|V|-\left\|f_{V}\right\|\right) \log _{2} N_{\pi}\left(L_{\pi}(f) / 4, n\right)\right)^{1 / 2} \\
& \leqslant\left(2(|D|-\|\bar{f}\|) \log _{2} N_{\pi}\left(L_{\pi}(f) / 2, n\right)\right)^{1 / 2}+\left(2\left(|V|-\left\|f_{V}\right\|\right) \log _{2} N_{\pi}\left(L_{\pi}(f) / 4, n\right)\right)^{1 / 2} \\
& \leqslant\left(2|D| \log _{2} N_{\pi}\left(L_{\pi}(f) / 2, n\right)\right)^{1 / 2}+\left(2|D| \log _{2} N_{\pi}\left(L_{\pi}(f) / 4, n\right)\right)^{1 / 2} \\
& \leqslant 2\left(2|D| \log _{2} N_{\pi}\left(L_{\pi}(f) / 2, n\right)\right)^{1 / 2} .
\end{aligned}
$$

Очевидно, что область $V^{\prime}$ удовлетворяет требуемым условиям. Лемма доказана. 
Доказательство теоремы 5 почти дословно повторяет доказательство теоремы 2. Поэтому приведем теорему 5 без доказательства.

Теорема 5. Пусть

$$
f:\{0,1\}^{n} \rightarrow\{0,1\}, \quad L(f) \geqslant c_{22} n^{3},
$$

где с $c_{22}$ - постоянжая. Тогда сущестөуют тахие область $D \subseteq\{0,1\}^{n}$ u постоянная $c_{23}$, чmo

$$
L_{\pi}\left(f_{D}\right) \geqslant n, \quad \frac{|D|}{\log _{2} N_{\pi}\left(L_{\pi}\left(f_{D}\right), n\right)} \leqslant c_{23} .
$$

\section{4. Сложность частичных и полностью определенных булевых функций}

Пусть $D \subseteq\{0,1)^{n},|D|<2^{n / 2}$. В [2] доказано существование такого линейного оператора

$$
F:\{0,1\}^{n} \rightarrow\{0,1\}^{2] \log _{2}|D| \mid}
$$

что для любых $x, y \in D, F(x) \neq F(y)$, если $x \neq y$. Воспользуемся әтим результатом. Пусть $f: D\{0,1\}$. Положим $m=2] \log _{2}|D|[$. Рассмотрим функцию $f^{\prime}:\{0,1\}^{m} \rightarrow\{0,1\}$ такую, что $f^{\prime}(z)=f(y)$, если $y \in D$ и $F(y)=z$. Схема, реализующая функцию $f$, может быть получена из схемы $S^{\prime}$, реализующей функцию $f^{\prime}$, если к входам $S^{\prime}$ подключить выходы схемы, реализующей оператор $F$. Поәтому очевидно, что

$$
L\left(f^{\prime}\right) \geqslant L(f)-L(F)
$$

Пусть

$$
|D|=n^{O(1)} \text {. }
$$

В этом случае нетрудно показать, что

$$
L(F)=O(n)
$$

Следовательно, доказательство невысокой нижней оценки сложности для функции $f$ влечет доказательство әкспоненциальной нижней оценки сложности для функции $f^{\prime}$. Действительно, если $L(f) \geqslant L(F)+\varepsilon n$, где $\varepsilon-$ некоторая постоянная, то

$$
L\left(f^{\prime}\left(z_{1}, \ldots, z_{m}\right)\right) \geqslant \varepsilon n=\varepsilon 2^{\log _{2} n}=\varepsilon 2^{O(m)} .
$$

Похожая ситуация имеет место и для других управляющих систем. Пусть $S^{\prime}$ - контактная схема, реализующая функцию $f^{\prime}$, и пусть $l_{i}\left(x_{1}, \ldots, x_{n}\right)-i$-я функция оператора $F$. Заменим в схеме $S^{\prime}$ каждый контакт вида $z_{i}^{\sigma_{i}}$ на контактную схему, реализующую функцию $z_{i}^{\sigma_{i}}\left(x_{1}, \ldots, x_{n}\right)$. Нетрудно убедиться в том, что новая схема $S$ будет реализовывать функцию $f$. Так как линейная функция от $n$ переменных реализуется контактной схемой со сложностью, не превосходящей $4 n-2$, то очевидно, что

$$
L_{\mathrm{kc}}\left(f^{\prime}\right) \geqslant \frac{L_{\mathbf{k c}}(f)}{4 n} .
$$


Если $L_{\text {кс }}(f) \geqslant 4 n^{1+\varepsilon}$, где $\varepsilon-$ положительная постоянная, то

$$
L_{\mathrm{\kappa c}}\left(f^{\prime}\left(z_{1}, \ldots, z_{m}\right)\right) \geqslant n^{\varepsilon}=2^{\varepsilon \log _{2} n}=2^{O(m)} .
$$

Подобный результат справедлив и для формул в базисе, содержащем линейную функцию. Легко видеть, что

$$
L_{\phi}\left(f^{\prime}\right) \geqslant \frac{L_{\phi}(f)}{n} .
$$

Следовательно, если $L_{\phi}(f) \geqslant n^{1+\varepsilon}$, то

$$
L_{\phi}\left(f^{\prime}\left(z_{1}, \ldots, z_{m}\right)\right) \geqslant n^{\varepsilon}=2^{O(m)} .
$$

Так как сложность реализации линейной функции $\pi$-схемами не более, чем в два раза превосходит квадрат числа переменных [3], то

$$
L_{\pi}\left(f^{\prime}\right) \geqslant \frac{L_{\pi}(f)}{2 n^{2}}
$$

Следовательно, если $L_{\pi}(f) \geqslant 2 n^{2+\varepsilon}$, то

$$
L_{\pi}\left(f^{\prime}\left(z_{1}, \ldots, z_{m}\right)\right) \geqslant n^{\varepsilon}=2^{O(m)} .
$$

Из (25)-(28) следует, что доказательство невысоких нижних оценок сложности частичных булевых функций с малой областью определения и доказательство әкспоненциальных нижних оценок сложности полностью определенных булевых функций являются в некотором смысле әквивалентными по трудности решения задачами. В связи с әтим можно высказать предположение, что существование у булевых функций сложных сужений на малые области является препятствием для доказательства относительно невысоких нижних оценок сложности. Это предположение на качественном уровне подтверждается известными в данный момент максимальными нижними оценками сложности реализации булевых функций схемами из функциональных әлементов, контактными схемами, формулами и $\pi$-схемами. С точностью до постоянных множителей әти оценки для некоторых функций $f_{1}, f_{2}, f_{3}, f_{4}$ имеют следующий вид [4]:

$$
\begin{aligned}
L\left(f_{1}\right) \geqslant n, & L_{\mathrm{kc}}\left(f_{2}\right) \geqslant \frac{n^{2}}{\log _{2} n}, \\
L_{\phi}\left(f_{3}\right) \geqslant \frac{n^{2}}{\log _{2} n}, & L_{\pi}\left(f_{4}\right) \geqslant n^{3} .
\end{aligned}
$$

С другой стороны, из теорем 1,4 и их аналогов для контактных схем и формул следует, что если сложности некоторых функций $g_{1}, g_{2}, g_{3}, g_{4}$ удовлетворяют неравенствам

$$
\begin{aligned}
L\left(g_{1}\right) & \geqslant c n^{1+\log _{2} 3}, & L_{\mathrm{kc}}\left(g_{2}\right) & \geqslant c n^{3+\varepsilon} \\
L_{\phi}\left(g_{3}\right) & \geqslant c n^{3+\varepsilon}, & L_{\pi}\left(g_{4}\right) & \geqslant c n^{4+\varepsilon}
\end{aligned}
$$


где $c$ и $\varepsilon$ - положительные постоянные, причем $\varepsilon$ может быть сколь угодно малой, то существуют такие области $D_{1}, D_{2}, D_{3}, D_{4} \subseteq\{0,1\}^{n}$, что $\left|D_{i}\right|=n^{a}$, где $i \in[1, \ldots, 4], a-$ постоянная,

$$
\begin{array}{rlrl}
L\left(g_{1} D_{1}\right) & \geqslant c^{\prime} n, & L_{\mathrm{kc}}\left(g_{2 D_{2}}\right) & \geqslant c^{\prime} n^{1+\varepsilon}, \\
L_{\phi}\left(g_{3 D_{3}}\right) & \geqslant c n^{1+\varepsilon}, & L_{\pi}\left(g_{4 D_{4}}\right) \geqslant c^{\prime} n^{2+\varepsilon},
\end{array}
$$

где $c^{\prime}$ - постоянная.

Сравнивая (31) с (25)-(28), замечаем, что выполнение неравенств (30) влечет существование у функций $g_{1}, g_{2}, g_{3}, g_{4}$ сужений, эквивалентных в некотором смысле экспоненциально сложным функциям. Легко видеть, что неравенства (29) и (30) определяют один и тот же порядок на множестве рассмотренных выше мер сложности булевых функций.

\section{5. Добавление}

Лемма 6. Пусть $a, b, c, d$ - матуральные числа,

$$
[\sqrt{2 a b}] \leqslant d \leqslant a / 2, \quad a>2 b>5 .
$$

Тогда махсимальное $c$, удовлетворяющее неравенству

$$
\left(\begin{array}{l}
a \\
d
\end{array}\right) /\left(\begin{array}{c}
a-c \\
d
\end{array}\right) \leqslant e^{b}
$$

не превосходит $d$.

Доказательство. Доказывать лемму будем от противного. Предположим, что максимальное $c$, удовлетворяющее неравенству (32), больше $d$. Неравенство (32) равносильно неравенству

$$
\ln \left(\left(\begin{array}{l}
a \\
d
\end{array}\right) /\left(\begin{array}{c}
a-c \\
d
\end{array}\right)\right)-b \leqslant 0 .
$$

Оценим снизу левую часть (33). Для этого воспользуемся хорошо известными неравенствами

$$
e^{t /(1+t)}<1+t<e^{t}
$$

справедливыми при $t>0$, и неравенствами

$$
\frac{n^{k}}{k !} e^{-k(k-1) /(2(n-k+1))} \leqslant\left(\begin{array}{l}
n \\
k
\end{array}\right) \leqslant \frac{n^{k}}{k !} e^{-k(k-1) /(2 n)} .
$$


Тогда

$\ln \left(\left(\begin{array}{l}a \\ d\end{array}\right) /\left(\begin{array}{c}a-c \\ d\end{array}\right)\right)-b \geqslant \ln \frac{a^{d} e^{-d(d-1) /(2(a-d+1))}}{(a-c)^{d} e^{-d(d-1) /(2(a-c))}}-b$

$$
\begin{aligned}
& =d \ln \left(1+\frac{c}{a-c}\right)+\frac{d(d-1)}{2} \frac{(c-d+1)}{(a-c)(a-d+1)}-b \\
& \geqslant d \frac{c}{a-c}+\frac{d(d-1)(c-d+1)}{2(a-c)(a-d+1)}-b \geqslant \frac{d^{2}}{a}-b \\
& \geqslant \frac{(\sqrt{2 a b}-1)^{2}}{a}-b>\frac{a b-2 \sqrt{2 a b}}{a}>\left(\frac{b}{a}\right)^{1 / 2}(\sqrt{a b}-\sqrt{8})>0 .
\end{aligned}
$$

Получили противоречие с (32). Лемма доказана.

Лемма 7. Пусть $1 \leqslant k \leqslant n$. Тогда

$$
\begin{aligned}
\log _{2} N((L-k+1) / k, n) & \leqslant \frac{1}{k} \log _{2} N(L, n)+\frac{1}{k} \\
\log _{2} N_{\pi}(L / k, n) & \leqslant \frac{1}{k} \log _{2} N_{\pi}(L, n)+\frac{1}{k} .
\end{aligned}
$$

Доказательство. Доказательства двух неравенств аналогичны. Поэтому докажем только первое из них. Нетрудно убедиться в справедливости неравенства

$$
\left(\begin{array}{c}
N(L, n) \\
k
\end{array}\right) k ! \leqslant N(k L+k-1, n) .
$$

А так как

$$
\left(\begin{array}{c}
N(L, n) \\
k
\end{array}\right) k ! \geqslant \frac{N(L, n)^{k}}{k !} k ! e^{-k(k-1) /(2(N(L, n)-k+1))} \geqslant \frac{N(L, n)^{k}}{2}
$$

To

$$
k \log _{2} N(L, n)-1 \leqslant \log _{2} N(k L+k-1, n) .
$$

Пусть $k L+k-1=L^{\prime}$. Тогда

$$
\log _{2} N\left(\left(L^{\prime}-k+1\right) / k, n\right) \leqslant \frac{1}{k} \log _{2} N\left(L^{\prime}, n\right)+\frac{1}{k}
$$

Лемма доказана.

Лемма 8. Сущестөует тахая постоянная $c_{14}$, что для лгбого $d, n^{1+\alpha} \leqslant d \leqslant 2^{n}$, любой области $D \in D(d)$ и любой фужкции $f: D \rightarrow\{0,1\} \cdot$ веса $n^{\alpha}$ справедливо неравенство

$$
L(f) \leqslant c_{14} \frac{n^{\alpha} \ln d}{\ln n} .
$$


Дохазательство. Утверждения леммы выводятся из следующих фактов.

Пусть $D \subseteq\{0,1\}^{n},|D|<2^{n / 2}$. Тогда существует такой линейный оператор

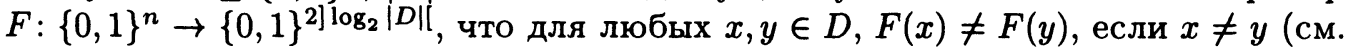
лемму 4 в [2]).

Пусть $f:\{0,1\}^{n} \rightarrow\{0,1\}, f>$ n. Тогда (см. теорему 3.4 в [1])

$$
L(f) \leqslant \frac{\ln \left(\begin{array}{c}
2^{n} \\
\|f\|
\end{array}\right)}{\ln \ln \left(\begin{array}{c}
2^{n} \\
\|f\|
\end{array}\right)}(1+o(1)) .
$$

Так как

$$
L(F) \leqslant \frac{8 n] \log _{2}|D|[}{\log _{2} n}=o\left(\frac{n^{\alpha} \ln |D|}{\ln n}\right)
$$

достаточно показать, что при некоторой постоянной $c_{14}$ справедливо неравенство

$$
\frac{\ln \left(\begin{array}{l}
d^{2} \\
n^{\alpha}
\end{array}\right)}{\ln \ln \left(\begin{array}{l}
d^{2} \\
n^{\alpha}
\end{array}\right)} \leqslant c_{14} \frac{n^{\alpha} \ln d}{\ln n} .
$$

Для доказательства последнего неравенства заметим, что величина, стоящая в его левой части, возрастает с увеличением $d, \mathrm{a}$

$$
\ln \left(\begin{array}{l}
d^{2} \\
n^{\alpha}
\end{array}\right) \leqslant \ln \frac{d^{2 n^{\alpha}}}{\left(n^{\alpha}\right) !} \leqslant 2 n^{\alpha} \ln d .
$$

Лемма доказана.

\section{Список литературы}

1. Лупанов О. Б. Об одном подходе к синтезу управляющих систем - принципе локального кодирования. Пробл. киберн. (1965) 14, 31-110.

2. Андреев А. Е. О сложности реализации частичных булевых функций схемами из функциональных әлементов. Дискретная математиха (1989) 1, №4, 36-45.

3. Яблонский С. В. Реализация линейной функции в классе $\pi$-схем. Докл. $A H C C C P$ (1954) 94, №5, 805-806.

4. Нечипорук Э. И. Об одной булевской функции. Докл. АН $C C C P$ (1966) 169, ㅅ4, 765767.

Статья поступила 21.06.94. 